\title{
In-medium transverse momentum broadening effects on di-jet observables
}

\section{Martin Rohrmoser, ${ }^{a}{ }^{*}$ Krzysztof Kutak, $^{a}$ Andreas van Hameren, ${ }^{a}$ Wiesław Płaczek ${ }^{b}$ and Konrad Tywoniuk}

${ }^{a}$ Institute of Nuclear Physics,

Polish Academy of Sciences, ul. Radzikowskiego 152, 31-342 Kraków, Poland

${ }^{b}$ Institute of Applied Computer Science, Jagiellonian University,

ul. Łojasiewicza 11, 30-348 Kraków, Poland

${ }^{c}$ Department of Physics and Technology,

University of Bergen, 5007 Bergen, Norway

E-mail: rohrmoser.martin1987@gmail.com, krzysztof.kutak@ifj.edu.pl, Andre.Hameren@ifj.edu.pl, Wieslaw.Placzek@uj.edu.pl,

Konrad.Tywoniuk@uib.no

Heavy ion collisions at high energies can be used as an interesting way to recreate and study the medium of the quark-gluon plasma (QGP). We particularly investigate the jets produced in hard binary collisions and their interactions with a tentative medium. These jets were obtained numerically from the Monte-Carlo simulations of hard collisions using the KATIE-algorithm [1], where parton momenta within the colliding nucleons were describe by means of unintegrated parton distribution functions (uPDF). We evolved these jets within a medium that contains both, transverse kicks (yielding a broadening in momentum transvers to the jet-axis) as well as medium induced radiation within the MINCAS-algorithm [2] following the works of [3, 4]. We produce qualitative results for the decorrelation of dijets. In particular, we study deviations from a transverse momentum broadening that follows a Gaussian distribution.

HardProbes 2020

1-6 June 2020

Austin, Texas

${ }^{*}$ Speaker 
We study jet-pairs produced in hard partonic collisions during the early stages of ultrarelativistic heavy ion collisions. In order to study the ultrarelativistic nuclear collisions, we first describe di-jet production in $p-p$ collisions, where we assume that a QGP-medium is not formed, and later on extend this description to include medium effects. In our study of di-jet observables we focus on distributions of jet-pairs in the transverse plane, orthogonal to the collision axis of the incoming nucleons. In this case the momentum components of the incoming hard partons transverse to the collision axis cannot be neglected, since they contribute considerably to the deviations of pure back-to-back emission of the di-jet pairs. Thus, we describe the di-jet production cross section $\sigma_{\text {hard }}$ using the following factorization formula

$$
\begin{aligned}
\frac{d \sigma_{\text {hard }}}{d y_{1} d y_{2} d^{2} q_{1 T} d^{2} q_{2 T}}= & \int \frac{d^{2} k_{1 T}}{\pi} \frac{d^{2} k_{2 T}}{\pi} \frac{1}{16 \pi^{2}\left(x_{1} x_{2} s\right)^{2}} \overline{\left|\mathcal{M}_{g^{*} g^{*} \rightarrow g g}^{\text {off-shll }}\right|^{2}} \\
& \times \delta^{2}\left(\vec{k}_{1 T}+\vec{k}_{2 T}-\vec{q}_{1 T}-\vec{q}_{2 T}\right) \mathcal{F}_{g}\left(x_{1}, k_{1 T}^{2}, \mu_{F}^{2}\right) \mathcal{F}_{g}\left(x_{2}, k_{2 T}^{2}, \mu_{F}^{2}\right),
\end{aligned}
$$

where $\mathcal{F}_{g}\left(x_{i}, k_{i T}^{2}, \mu_{F}^{2}\right)$ (for $\left.i=1,2\right)$ are unintegrated parton distribution functions at factorization scale $\mu_{F}$, which give the distribution of the transverse momenta $k_{i T}$ and the momentum fraction $x_{i}$ in direction of the collision axis. The momentum fractions $x_{i}$, the rapidities $y_{i}$ and the transverse momenta $q_{i T}$ of the outgoing particles are related to one another as $x_{1}=\frac{q_{1 T}}{\sqrt{s}} \exp \left(y_{1}\right)+\frac{q_{2 T}}{\sqrt{s}} \exp \left(y_{2}\right)$, and $x_{2}=\frac{q_{1 T}}{\sqrt{s}} \exp \left(-y_{1}\right)+\frac{q_{2 T}}{\sqrt{s}} \exp \left(-y_{2}\right) \cdot \mathcal{M}_{g^{*} g^{*} \rightarrow g \bar{g}}^{\text {off-shell }}$ is the matrix element for the collision of virtual incoming particles and outgoing particles that are on the mass shell. We obtained our numerical results for $p$ - $p$ collisions via an implementation of Eq. (1) in the Monte-Carlo program KATIE.

For the description of di-jet production in heavy ion collisions, it can be approximated that the probability densities for di-jet production in heavy-ion collisions factorizes into a density for the initial hard scattering and a density for the jet-medium interactions, since either of these subprocesses involves largely different scales of momentum transfer. We again describe the hard scattering using the factorization formula Eq. (1). We consider an in-medium jet-evolution for which, under the assumption that transverse momentum transfers from the medium to the jet particles are small, the following evolution equation was found in [5] for distributions of the leading jet particle

$$
\begin{aligned}
\frac{\partial}{\partial t} D(\tilde{x}, \mathbf{l}, t) & =\frac{1}{t^{*}} \int_{0}^{1} d z \mathcal{K}(z)\left[\frac{1}{z^{2}} \sqrt{\frac{z}{\tilde{x}}} D\left(\frac{\tilde{x}}{z}, \frac{\mathbf{l}}{z}, t\right) \theta(z-\tilde{x})-\frac{z}{\sqrt{\tilde{x}}} D(\tilde{x}, \mathbf{l}, t)\right] \\
& +\int \frac{d^{2} \mathbf{q}}{(2 \pi)^{2}} C(\mathbf{q}) D(\tilde{x}, \mathbf{l}-\mathbf{q}, t),
\end{aligned}
$$

with $\frac{1}{t^{*}}=\frac{\alpha_{s} N_{c}}{\pi} \sqrt{\frac{\hat{q}}{E}}$ defined via the quenching parameter $\hat{q}$ and the energy of the incoming parton E. $D(\tilde{x}, \mathbf{l}, t)$ is defined as an energy density with $\tilde{x}$ the fraction of energy $E$ that the jet-particle retains and $\mathbf{l}$ its momentum component orthogonal to the momentum of the incoming particle. The splitting kernel takes the form

$$
\mathcal{K}(z)=\frac{[f(z)]^{5 / 2}}{[z(1-z)]^{3 / 2}}, \quad f(z)=1-z+z^{2}, \quad 0 \leq z \leq 1,
$$

The scattering kernel is

$$
C(\mathbf{q})=w(\mathbf{q})-\delta(\mathbf{q}) \int d^{2} \mathbf{q}^{\prime} w\left(\mathbf{q}^{\prime}\right)
$$


We consider here a scattering off medium particles of a form [6] such that

$$
w(\mathbf{q})=\frac{16 \pi^{2} \alpha_{s}^{2} N_{c} n}{\mathbf{q}^{2}\left(\mathbf{q}^{2}+m_{D}^{2}\right)},
$$

with $m_{D}$ the medium quasi-particle Debye mass. After integration of Eq. (2) over the transverse momenta, one obtains

$$
\frac{\partial}{\partial t} D(\tilde{x}, t)=\frac{1}{t^{*}} \int_{0}^{1} d z \mathcal{K}(z)\left[\sqrt{\frac{z}{\tilde{x}}} D\left(\frac{\tilde{x}}{z}, t\right) \theta(z-\tilde{x})-\frac{z}{\sqrt{\tilde{x}}} D(\tilde{x}, t)\right],
$$

It was shown in [2] that both Eqs. (2) and (6) can be written as integral equations, which can be solved numerically via a Monte-Carlo algorithm, as it was done by the MINCAS-program [2].

In our approach [7] we obtained the four momenta of the gluons produced in the hard collisions via the KATIE-program and then the changes in gluon momenta due to in-medium evolution via the MINCAS-program. We parametrize the medium as follows: $\hat{q}=0.29 \mathrm{GeV}^{2} / \mathrm{fm}, n=0.08 \mathrm{GeV}^{3}$, $m_{D}=0.61 \mathrm{GeV}$. These parameters are estimates for a medium of constant temperature $T=250 \mathrm{MeV}$ (cf. [7] for further explanations). The particles evolve over a time of $t_{L}=5 \mathrm{fm} / \mathrm{c}$ in the medium.

We obtained numerical results for the azimuthal angular decorrelations $\frac{d N}{d \Delta \phi}$ ( $N$ is the number of di-jets; $\Delta \phi$ is the difference of the azimuthal angles of the two outgoing jet-momenta). We compared the following three cases

1. A case without jet-medium interactions, referred to as the "vacuum case", where the outgoing di-jet momenta where obtained via the KATIE-algorithm

2. A case with jet-medium interactions, where the in-medium jet-evolution follows Eq. (2). We refer to this case as "non-Gaussian $k_{T}$ broadening".

3. A case referred to as "Gaussian $k_{T}$ broadening", where the distribution of momentum fractions $\tilde{x}$ follow Eq. (6), while the transverse momentum components $\mathbf{I}$ are selected from a Gaussian distribution $P(|| \mathbf{I}||)$, i.e.: $P(|| \mathbf{I}||)=\frac{1}{\sqrt{2 \pi \hat{q} t_{L}}} \exp \left(-\frac{\mathbf{l}^{2}}{2 \hat{q} t_{L}}\right)$.

For jet-pairs produced in collisions at $\sqrt{s_{\mathrm{NN}}}=2.76 \mathrm{TeV}$, where jets are emitted in directions with rapidities $1.2<|y|<2.1$, the left panel of Fig. 1 shows results for $\frac{d N}{d \Delta \phi}$. There, the cases with jetmedium interactions are considerably suppressed. Differences in shapes of the cases are examined in the right panel of Fig. 1, which shows the $\frac{d N}{d \Delta \phi}$ distributions for all three cases divided by their respective maxima. The vacuum case behaves similarly to the case of Gaussian $k_{T}$ broadening, where the curves for the latter case correspond to an even slightly narrower distribution than that of the former. However, the distribution for non-Gaussian $k_{T}$ broadening is considerably broader.

We studied di-jet production in heavy ion collisions by using a Monte-Carlo approach which accounts for the momentum components of the colliding partons transverse to the beam axis via unintegrated parton densities and an in-medium jet evolution that follows Eqs. (2) and (6) from [5] with both scattering off-medium particles and coherent medium induced radiation. Some shortcomings of our approach are that it does not include quark jets, that the leading jet-gluon momenta are taken as the jet momenta, and that we do not include bremsstrahlung emission in vacuum. Thus, we do not use our approach for comparison with the experiment, but rather to qualitatively study the influences of in-medium interactions on di-jet observables. 

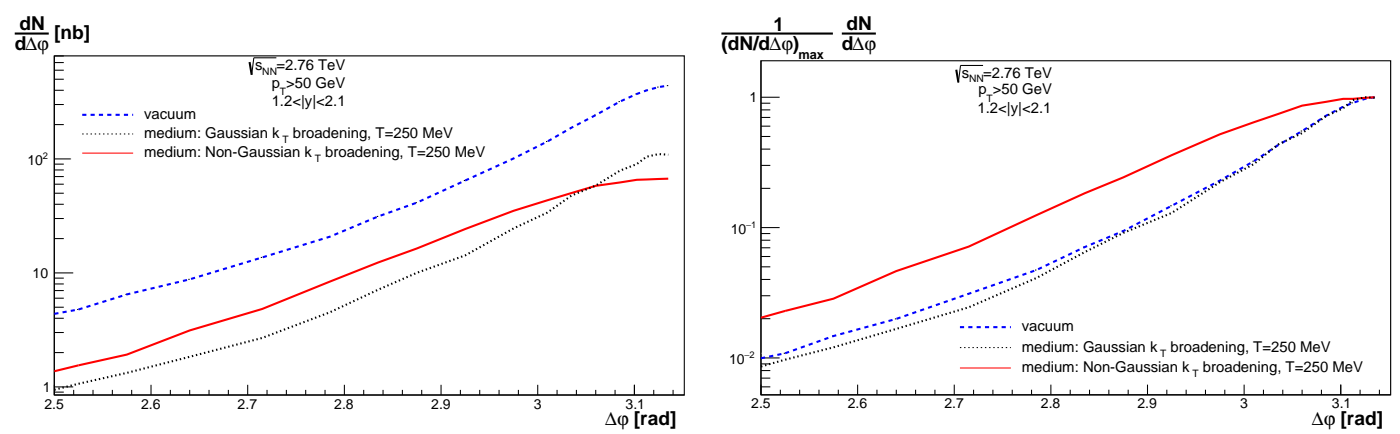

Figure 1: Left panel: results for azimuthal angular decorrelations for di-jets produced in nuclear collisions at $\sqrt{s_{\mathrm{NN}}}=2.76 \mathrm{TeV}$. Right panel: same curves, but now normalized to their respective maxima.

We obtained phenomenological results for the azimuthal angular decorrelations $\frac{d N}{d \Delta \phi}$. We conclude that the obtained distribution for di-jets in a QGP-medium is considerably suppressed as compared to di-jet production without consideration of a medium and also considerably broader. The main reason of the broadening are deviations of the $k_{T}$ distributions from Gaussian behavior.

\section{Acknowledgements}

The research is supported by Polish National Science Centre grant no. DEC-2017/27/B/ST2/01985

\section{References}

[1] A. van Hameren, KaTie : For parton-level event generation with $k_{T}$-dependent initial states, Comput. Phys. Commun. 224 (2018) 371 [1611.00680].

[2] K. Kutak, W. Płaczek and R. Straka, Solutions of evolution equations for medium-induced QCD cascades, Eur. Phys. J. C79 (2019) 317 [1811.06390].

[3] J.-P. Blaizot, F. Dominguez, E. Iancu and Y. Mehtar-Tani, Medium-induced gluon branching, JHEP 01 (2013) 143 [1209.4585].

[4] J.-P. Blaizot, F. Dominguez, E. Iancu and Y. Mehtar-Tani, Probabilistic picture for medium-induced jet evolution, JHEP 06 (2014) 075 [1311. 5823].

[5] J.-P. Blaizot, L. Fister and Y. Mehtar-Tani, Angular distribution of medium-induced QCD cascades, Nucl. Phys. A940 (2015) 67 [1409.6202].

[6] P. Aurenche, F. Gelis and H. Zaraket, A Simple sum rule for the thermal gluon spectral function and applications, JHEP 05 (2002) 043 [hep-ph/0204146].

[7] A. van Hameren, K. Kutak, W. Płaczek, M. Rohrmoser and K. Tywoniuk, Jet quenching and effects of non-Gaussian transverse-momentum broadening on di-jet observables, 1911.05463. 